\title{
Dissipation and enstrophy statistics in turbulence: are the simulations and mathematics converging?
}

\author{
R. M. Kerr $\dagger$ \\ School of Engineering, University of Warwick, \\ Coventry CV4 7AL, UK
}

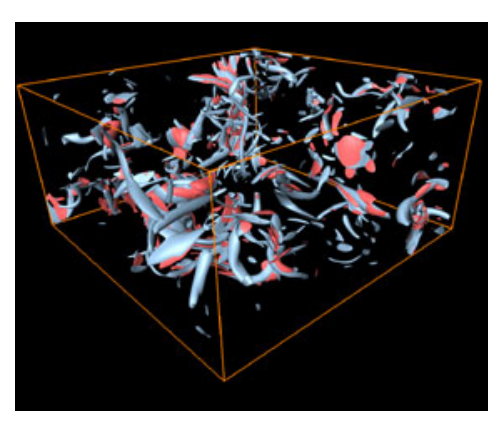

\begin{abstract}
Since the advent of cluster computing over 10 years ago there has been a steady output of new and better direct numerical simulation of homogeneous, isotropic turbulence with spectra and lower-order statistics converging to experiments and many phenomenological models. The next step is to directly compare these simulations to new models and new mathematics, employing the simulated data sets in novel ways, especially when experimental results do not exist or are poorly converged. For example, many of the higher-order moments predicted by the models converge slowly in experiments. The solution with a simulation is to do what an experiment cannot. The calculation and analysis of Yeung, Donzis \& Sreenivasan (J. Fluid Mech., this issue, vol. 700, 2012, pp. 5-15) represents the vanguard of new simulations and new numerical analysis that will fill this gap. Where individual higher-order moments of the vorticity squared (the enstrophy) and kinetic energy dissipation might be converging slowly, they have focused upon ratios between different moments that have better convergence properties. This allows them to more fully explore the statistical distributions that eventually must be modelled. This approach is consistent with recent mathematics that focuses upon temporal intermittency rather than spatial intermittency. The principle is that when the flow is nearly singular, during 'bad' phases, when global properties can go up and down by many orders of magnitude, if appropriate ratios are taken, convergence rates should improve. Furthermore, in future analysis it might be possible to use these ratios to gain new insights into the intermittency and regularity properties of the underlying equations.
\end{abstract}

\section{Introduction}

Since 1972, direct three-dimensional simulations of the incompressible Navier-Stokes equations have been providing insights into the statistical properties of turbulence. Even the first calculation of Orszag \& Patterson (1972) was able to reproduce the simplest third-order moment of the velocity derivative, the velocity derivative skewness $S_{3}$, obtained experimentally from hot-wire anemometers. That is, for

$$
p=3: \quad-S_{p}=-\overline{(\partial u / \partial x)^{p}} /\left(\overline{(\partial u / \partial x)^{2}}\right)^{p / 2} \sim 0.4-0.5 .
$$

$\dagger$ Email address for correspondence: R.M.Kerr@warwick.ac.uk 
This value showed that the rate of enstrophy production (production of the meansquare vorticity) was in the fully developed turbulent regime, a clear sign that these simulations were generating a turbulent flow, even if the simulations were far from the regime needed to see a $k^{-5 / 3}$ Kolmogorov inertial subrange.

This calculated skewness is only one example of the role that moments of the dissipation and vorticity fields play in our understanding of the statistics and structure of turbulence. Furthermore, mathematics of the Navier-Stokes equations now provides some precise bounds for some of these and with recent advances in computing power, the time has come to make some direct comparisons between this mathematics and high-Reynolds-number numerical data. One goal of these comparisons will be to provide insight into further mathematics that should eventually lead us to a solution of the Navier-Stokes question, as posed by the Clay Foundation (Clay 2012), which is: Are these equations well-posed mathematically, without singularities, or do they have unphysical singularities?

The mathematical questions usually address the vorticity, but what an engineer needs for modelling are the strength and location of the kinetic energy dissipation, the associated strain field, fluxes and the role of pressure gradients. What can simulations tell us here? Accomplishing these goals will require more than the largest highestReynolds-number calculations, it will also require innovative use of those results in ways suggested by the latest mathematical results. The paper being highlighted (Yeung, Donzis \& Sreenivasan 2012) represents a first step in that direction.

\section{Overview}

Besides the skewness, another early observation (Siggia 1981) was that vorticity was largely concentrated in vortex tubes, not vortex sheets. This concentration of vorticity is one manifestation of intermittency, the tendency for turbulent flows to have patches of intense fluctuations separated by quiescent regions. The spatial and statistical relationships between vorticity and the dissipation rate were then investigated. In the first detailed analysis of the strain, both its structure and its higher-order statistics (Kerr 1985), it was clearly demonstrated that the most intense dissipative/strain structures were sheets, not tubes, as seen in the figure by the title (Schumacher, Eckhardt \& Doering 2010, reproduced with permission) with vorticity isosurfaces in blue and dissipation in red. Dissipation was concentrated either around the vortex structures, or where vortex tubes were interacting most strongly. Overall, the higher-order strain moments were less intermittent than those of vorticity. The highest-order $(m>3)$ statistics converged very slowly and were not included.

In the following discussion, the single-times moments of the enstrophy will be denoted as $\left\langle\Omega^{m}\right\rangle=\overline{\omega^{2 m}}$ and of the dissipation by $\left\langle\epsilon^{m}\right\rangle=(2 v)^{m} \overline{\left(S_{i j} S_{i j}\right)^{m}}$, where the vorticity is $\boldsymbol{\omega}=\boldsymbol{\nabla} \times \boldsymbol{u}$ and the strain rate is $S_{i j}=(1 / 2)\left(\partial u_{i} / \partial x_{j}+\partial u_{j} / \partial x_{i}\right)$. In addition, $\left\langle\Omega^{\prime m}\right\rangle=\left\langle\Omega^{m}\right\rangle /(\langle\Omega\rangle)^{m}$, and similarly for $\left\langle\epsilon^{\prime m}\right\rangle$. Finally, curly brackets $\{\cdot\}$ indicate ensemble (that is time) averages.

The conclusion that enstrophy might be more intermittent than the dissipation was qualitatively confirmed by the next set of direct numerical simulation (DNS) of homogeneous, isotropic turbulence (Chen et al. 1997) using both moments and structure-function exponents, even though these results were inconsistent (at larger scales) with both the experiments and statistical arguments (Nelkin 1999; Kerr, Meneguzzi \& Gotoh 2001) available at that time. Ultimately, the only way to resolve this discrepancy was to do bigger and better numerical calculations. 

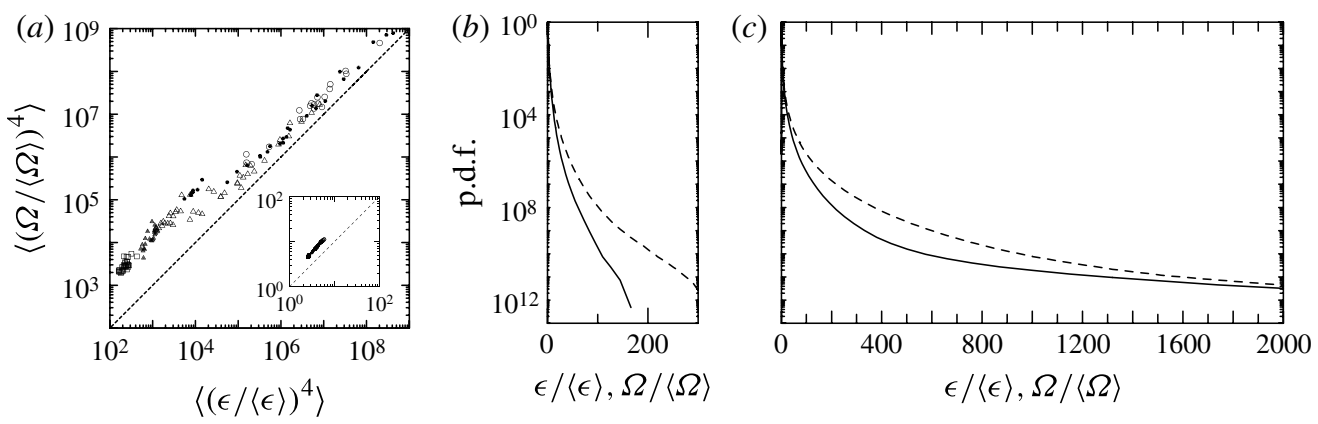

FIGURE 1. (a) Data on spatially-averaged moments of normalized dissipation and enstrophy for each snapshot, with different symbols for five simulations at $R_{\lambda}=140,240,380,600$ and 1000: $\square, \boldsymbol{\Delta}, \Delta, \bullet$ and $\circ$, respectively. The moments are plotted against each other (inset shows second moments). Note that despite many orders of magnitude in the range, the ratios of these fourth-order moments are nearly proportional to each other. P.d.f.s of normalized dissipation (solid lines) and enstrophy density (dashed lines) from two $4096^{3}$ simulations. $(b) R_{\lambda} \approx 240$, $k_{\max } \eta \approx 11 ;(c) R_{\lambda} \approx 1000, k_{\max } \eta \approx 1.4$. (Figure adapted from Yeung et al. 2012.)

These started to arrive with the use of cluster computing. Over the past 10 years there has been a steady output of new and better DNS of homogeneous, isotropic turbulence, largely summarized by Ishihara, Gotoh \& Kaneda (2009). Results include improved numerical spectra, structure function exponents and (up to order $\left\langle\epsilon^{2}\right\rangle$ and $\left\langle\Omega^{2}\right\rangle$ ), are consistent with the phenomenology that at sufficiently high Reynolds numbers the scaling properties of $\epsilon$ and $\Omega$ should converge.

However, what has not been completely answered is whether the higher-order longitudinal and transverse structure-function exponents converge and whether the higher-order dissipation $\langle\epsilon\rangle$ and enstrophy $\langle\Omega\rangle$ moments converge. Unfortunately, while it is the higher-order enstrophy moments that are needed for comparing with mathematical bounds, these have the poorest convergence properties.

Yeung et al. (2012) begins by addressing the poor convergence properties at higher orders. The solution is in how ratios of these moments of dissipation and enstrophy are distributed in time, shown in figure $1 a$ (from Yeung et al. 2012). This shows that their magnitudes are strongly correlated over a very wide range of scales. As a result, the ensemble average of the ratios converges, even when the individual moments do not. Once good statistics were available, Yeung et al. (2012) considers whether these ratios change as the Reynolds number increases. What is found is that at the highest $R_{\lambda}$, the ratio of $\left\{\left\langle\Omega^{\prime m}\right\rangle /\left\langle\epsilon^{\prime m}\right\rangle\right\}$ decreases between $m=3$ and $m=4$, whereas at lower Reynolds number it does not. Why?

The answer to this question is shown in figures $1 b-c$ (also from Yeung et al. 2012) which compares the tails of the two distributions at two Reynolds numbers. For $R_{\lambda}=240$ the tails do not converge, but for $R_{\lambda}=1000$ they do, even though the magnitudes of the dissipation and enstrophy flatness factors still differ due to the differences in the middle portion of the distributions.

These results seem to resolve whether dissipation and enstrophy statistics converge. Leaving us with: Why are the statistics of dissipation and enstrophy converging and why do the ratio statistics converge more rapidly? The answer to both might lie in new mathematical results on temporal intermittency, which could be tested numerically. 


\section{Future}

The underlying principle of the new mathematics for temporal intermittency is that there will be 'bad' regions with structures that could be reconnections, near singularities of the inviscid equations, or multi-fractal events. These events might be sparse, but their intensity can be bounded rigorously (Gibbon 2009). For example, it can be shown that

$$
\left\{\left\langle\Omega^{m}\right\rangle^{1 /(4 m-3)}\right\} \leqslant c_{m_{1}} v^{2 m /(4 m-3)} L^{-1} R e^{3},
$$

where $v$ is the viscosity and $R e$ is the Reynolds number.

The details of these bounds are not important here. What is important is that the bounds for different orders are related to the forcing in a well-defined way, and therefore, with the appropriate power-law scaling, these moments could be ordered in the manner predicted by these bounds. This is similar to how the higher moments of dissipation and enstrophy converge in Yeung et al. (2012), with even more hints of this ordering in the magnitudes of the moments in their table 2. What we need is more and better statistics like this. It is likely that this ordering is connected with a well-known upper bound for the growth of enstrophy in the Navier-Stokes equations $\langle\dot{\Omega}\rangle \leqslant C\langle\Omega\rangle^{3} / \nu^{3}$, which could be identified by short periods during which $\langle\Omega\rangle \leqslant C v^{3 / 2} /\left(T_{c}-t\right)^{1 / 2}$ (Schumacher et al. 2010).

Simulations such as Yeung et al. (2012) could test these possibilities, which suggests that we could be on the verge of answering some of the important questions about the scaling and intermittency properties of turbulence and hopefully gaining new insight into how to solve the regularity question for the Navier-Stokes equations.

\section{References}

Chen, S., Sreenivasan, K. R., Nelkin, M. \& CaO, N. 1997 Refined similarity hypothesis for transverse structure functions in fluid turbulence. Phys. Rev. Lett. 79, 2253-2256.

Clay Mathematical Institute, AMS-Clay Prize: www.claymath.org/millennium/.

GibBon, J. D. 2009 Estimating intermittency in three-dimensional Navier-Stokes turbulence. J. Fluid Mech. 625, 125-133.

Ishihara, T., Gotoh, T. \& KanedA, Y. 2009 Study of high-Reynolds number isotropic turbulence by direct numerical simulation. Annu. Rev. Fluid Mech. 41, 165-180.

KERR, R. M. 1985 Higher-order derivative correlations and the alignment of small-scale structures in isotropic numerical turbulence. J. Fluid Mech. 153, 31-58.

Kerr, R. M., Meneguzzi, M. \& Gotoh, T. 2001 An inertial range crossover in structure functions. Phys. Fluids 13, 1985-1994.

NELKIN, M. 1999 Enstrophy and dissipation must have the same scaling exponent in the high Reynolds number limit of fluid turbulence. Phys. Fluids 11, 2202-2204.

Orszag, S. A. \& Patterson, G. S. 1972 Numerical simulation of three-dimensional homogeneous isotropic turbulence. Phys. Rev. Lett. 28, 76-79.

SCHUMACHER, J., ECKHARDt, B. \& DOERING, C. R. 2010 Extreme vorticity growth in Navier-Stokes turbulence. Phys. Lett. A 374, 861-865.

SigGiA, E. D. 1981 Numerical study of small-scale intermittency in three-dimensional turbulence. J. Fluid Mech. 107, 375-406.

Vincent, A. \& Meneguzzi, M. 1991 The spatial structure and statistical properties of homogeneous turbulence. J. Fluid Mech. 225, 1-25.

Yeung, P. K., DonzIs, D. A. \& SReEnivasAn, K. R. 2012 Dissipation, enstrophy and pressure statistics in turbulence simulations at high Reynolds numbers. J. Fluid Mech. 700, 5-15. 